\title{
Quantitative assessment of the posttreatment lung volume changes in patients with flail chest on computed chest tomography images
}

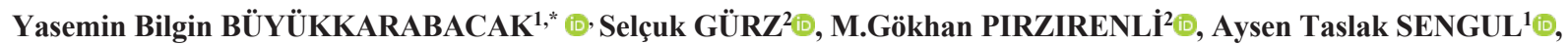 \\ Ahmet BASOGLU' ${ }^{1}$, Bunyamin SAHIN²
}

${ }^{1}$ Ondokuz Mayis University, Faculty of Medicine, Department of Thoracic Surgery, Atakum, Samsun, Turkey

${ }^{2}$ Ondokuz Mayis University, Faculty of Medicine, Department of Anatomy, Atakum, Samsun, Turkey

\begin{abstract}
Complications encountered in the chronic phase in flail chest patients are a decrease in lung volume due to chest wall deformities and secondary restrictive respiratory function disorders. The aim of the study was to determine the differences in lung volume changes between flail chest patients who were treated non-surgically and those who were treated with surgical stabilisation. The study was conducted on twenty patients who applied to our clinic and were diagnosed with flail chest. There were ten patients in the non-surgically treated group and ten in the surgical stabilisation group. Computed tomography (CT) images of the patients were received before the treatment and in the third post-operative month. Total lung volumes were estimated on CT images using the planimetry method of the Cavalieri principle. The relationship between the treatment method and volume changes was analysed. While the total lung volume increased $12.9 \%$ post-operatively in the non-surgically treated group, it increased $36.9 \%$ in the surgical stabilisation group $(\mathrm{p}<0.05)$. In those cases of hemithorax affected by trauma, the increase in volume in the surgical stabilisation group was $41.6(34.6 \%)$, but it was $7.7(10 \%)$ in the non-surgically treated group ( $<<0.05)$. In the contralateral hemithorax, the increase observed in the stabilisation group was 42.6 (39.2\%) while the increase in the non-surgical treated group was 19.6 (13.9\%) ( $>>0.05)$. Our findings showed that the post-operative total lung volume increase in the surgically treated group of patients was greater than that of the conservative group. If there are no preventive factors for the surgical approach, surgical rib stabilisation could be the primary choice of treatment in flail chest patients.
\end{abstract}

Keywords: Cavalieri principle, computed tomography, flail chest, pulmonary function, stereology, total lung volume

\section{Introduction}

When two or more adjacent ribs are broken in two or more sites and/or when at least two costochondral joint separations accompany a total sternum fracture, an incomplete and unstable segment is produced which causes paradoxical motion. This clinical condition is called flail chest. (Ahmed and Mohyuddin, 1995; Althausen et al, 2011; Fokin et al., 2020). The most important effects of flail chest in the chronic phase are a decrease in the lung volume due to chest wall deformities and secondary restrictive respiratory disorders (Athanassiadi et al., 2004). The chest wall deformities that can develop in the chronic period and the volume decrease in the chest wall secondary to the deformity can be prevented by chest wall stabilization. However, the results of pulmonary function tests can be affected by many conditions (pain, psychological/mentional condition etc.) in trauma patients. For this reason, pulmonary function tests (PFT) values cannot reflect no actual values about chest wall /pulmonary volume in flail chest patients after chest wall stabilization. Changes in the thorax/pulmonary volume of patients can be evaluated by steorological methods which could be evaluated independently of all of these conditions (Athanassiadi et al., 2004; Mehrabi et al., 2018).

Current stereological methods provide accurate assessment of the volumes of structures or organs through cross-sectional radiological images (Mehrabi et al., 2018). In recent years, studies assessing the lung volume through thoracic CT images have become widespread (Bulger et al., 
2000; Basoglu et al., 2007). Although there have been studies comparing the PFT of the flail chest patients who were treated non-surgically with those who underwent surgical stabilization, no studies have been encountered on the assessment of lung volume (Fabbri et al.,1996; Granetzny et al., 2005).

Our study aimed to compare the total lung volume of the flail chest patients who were treated non-surgically with that of the patients who underwent surgical stabilization. Depending on the quantitative results of the study, we tried to recommend the best approach for the treatment of the flail chest pathology.

\section{Material and method}

This study was approved by the local ethics committee of the Ondokuz Mayıs University. The patients who were treated and followed for flail chest were studied prospectively. Patients who applied to our clinic due to trauma and who had, unilaterally or bilaterally, three or more adjacent broken ribs in two or more regions and/or at least two costochondral joint separations accompanying a total sternum fracture were included in the study. The patients were studied for age, the number of rib fractures, the localization of flail chest, and preoperative and post-operative lung and lung volume changes.

18 were male and two were female patients included to the study. The mean age was 56.5 years (range 41-72). Ten patients were in the group without surgery and ten were in the surgical stabilization group. Both groups were similar in age, number of rib fractures, and location of flail chest. General features of the patients are given in the Table 1.

All patients were multidisciplinary followed in the intensive care unit. Patients whose blood gas values were $\mathrm{PaO} 2<60 \mathrm{mmHg}, \mathrm{PaCO} 245 \mathrm{mmHg}, \mathrm{PaO} 2$ sat $<85$, respiratory rate $>30 \mathrm{rate} / \mathrm{min}$ and/or who had a large flail chest on the anterolateral thoracic wall which disrupted the respiratory mechanics were intubated. The others were followed with $\mathrm{O} 2$ inhalation, postural drainage, nasotracheal and/or bronchoscopic aspiration if necessary, intense parenteral analgesics, and mucolytic and systemic antibiotic therapy. All patients underwent pulmonary physiotherapy.

Stabilization indications were (1) a large anterolateral flail chest (2) flail chest area causing paradoxical respiration (3) flail chest requiring mechanical ventilation support (4) pain and non-union rib fractures (5) thoracotomy due to intrathoracic hematoma drainage and decortications

Stabilization was performed only on the ribs which generated the paradoxical movement. The stabilization systems used for this purpose were U-shaped locking plates, straight plates, and straight-cornered plates. First the plate was shaped to fit to the concave structure of the rib. Then the rib was deperiosted and the plate was fixed on both sides of the fracture with at least two cortical screws. During stabilization, great attention must be paid to the protection of the intercostal neurovascular compartment. For this reason, particularly in patients where we have employed a U-shaped plaque, we prefer deperiosting the fractured rib and placing the stabilizer beneath the periosteum without causing any harm to the intercostal neurovascular bundle. In five patients three ribs, in two patients two ribs, in two patients four ribs and in one patient five ribs were stabilized. In patients with costochondral separation, the distal end of the plate was fixed to the nondamaged bone rib, and the proximal end was fixed to the corpus of the sternum through a tunnel opened under the pectoral muscle. In addition to displaced ribs, in one patient the sternum and in another patient a clavicle were also repaired.

All patients underwent computed thorax tomography (CTT) and pulmonary function tests (PFT) before the treatment and three months afterwards. Due to the patients' general condition, pulmonary functions could not be performed before the treatment.

The CTT was performed under a high-resolution scanner (Aquilion, Toshiba, Tochigi, Japan) with contrast medium. The scans were acquired during the inhalation phase of respiration with the patients in a supine position. The axial plane CTT scans were obtained with the following parameters: image size of $512 \times 512$, DFOV of 400 , KVP of 120 and slice thickness of $5 \mathrm{~mm}$ without intervals.

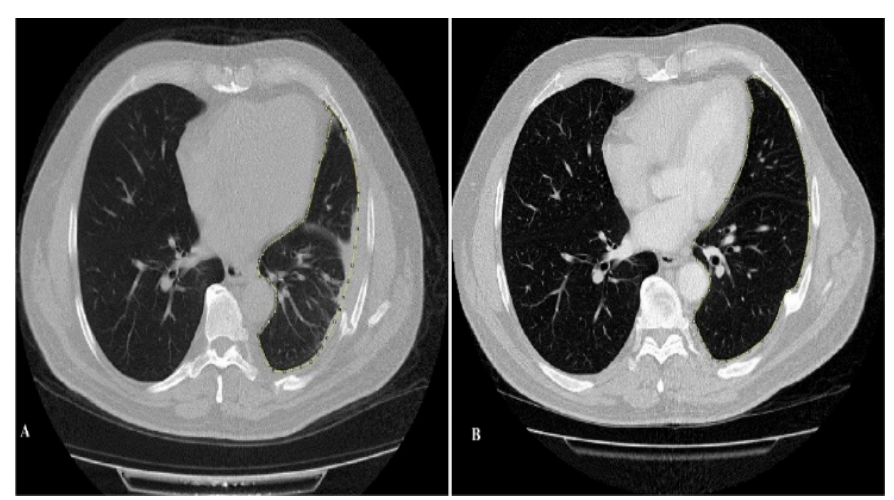

Fig. 1. An X-ray of postoperative patient with the repair of clavicle and ribs on the right side.

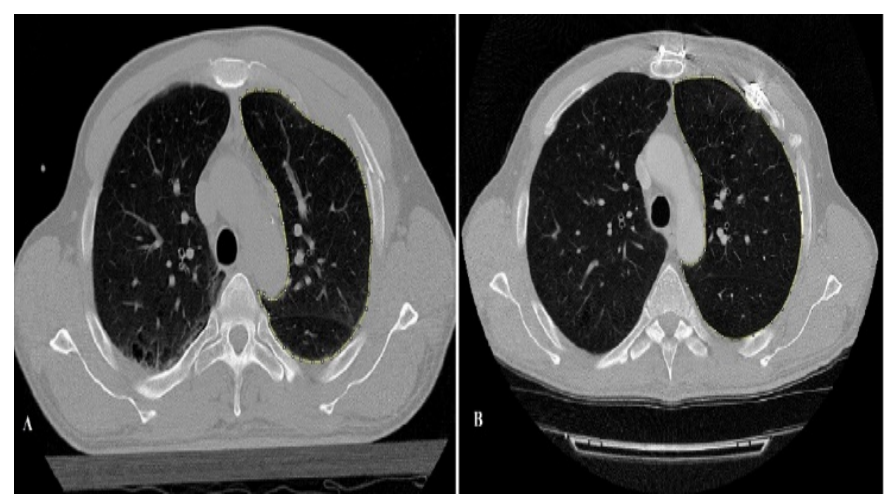

Fig. 2. Delineation of the lung boundaries during inspiration to measure the surface areas of the lung volume using the planimetry method in a patient. A: CCT section of the patient in admission. B: CCT section of the same patient in the third month.

Pre- and post-operative/control lung volumes were estimated using the Cavalieri principle of the stereological 
method on CTT images. One in every four slices was evaluated on the basis of systematic random sampling; therefore, sections with two-cm sampling intervals were used (Şahin et al.,2003a; Şahin et al.,2003b; Sahin and Ergur,2006; Konstantinov et al.,2009; Mazonakis et al., 2011) Sectional images recorded in Digital Imaging and Communications in Medicine (DICOM) format were viewed with the Onis program (free software; Digitalcore Co. Ltd.). The thicknesses of each slice as well as other information were noted. The images were exported from the software as separate DICOM files. Then, the images were opened in ImageJ software, which is distributed by the National Institutes of Health of the USA. After section sampling, the cut surface areas of the sections were automatically and semi-automatically measured using the software's threshold and manual planimetry options. The surface areas of the right and left lungs were measured separately in both groups' patients (Figure 1 and 2). The total volume was determined by multiplying the calculated section surface areas with the intervals between the sections (i.e., two $\mathrm{cm})$. All calculations were performed using Microsoft Excel. The coefficient of the error (CE) was also estimated for the all subjects depending on the given formula before.

Table 1. General Features of the patients

\begin{tabular}{|l|c|c|}
\hline \multicolumn{2}{|c|}{$\begin{array}{c}\text { Stabilisation } \\
\text { Group }\end{array}$} & $\begin{array}{c}\text { Non-surgical } \\
\text { Group }\end{array}$ \\
\hline $\begin{array}{l}\text { Number of } \\
\text { patients }\end{array}$ & 10 & 10 \\
\hline Age & $56.7 \pm 11.0$ & $49.8 \pm 17.6$ \\
\hline Gender & $10 \mathrm{M}$ & $8 \mathrm{M}, 2 \mathrm{~F}$ \\
\hline Height & $169.1 \pm 7.6$ & $170.2 \pm 11.3$ \\
\hline Weight & $72.6 \pm 14.2$ & $73.5 \pm 10.8$ \\
\hline $\begin{array}{l}\text { Average } \\
\text { number of } \\
\text { fractured ribs }\end{array}$ & $6.3 \pm 1.64$ & $5.9 \pm 1.52$ \\
\hline $\begin{array}{l}\text { Sternum } \\
\text { fracture }\end{array}$ & 1 & 1 \\
\hline $\begin{array}{l}\text { Costochondral } \\
\text { separation }\end{array}$ & 2 & 2 \\
\hline
\end{tabular}

\subsection{Statistical analysis}

The normality assumptions were checked using the ShapiroWilk test for continuous variables. The ages, weights, and heights of the patients were distributed normally for both groups. Since it was considered that the height of the patient would have an effect on the lung volume, a covariance analysis (ANCOVA) was employed in the analysis of the differences of the pre-operative and post-operative values to obtain an analysis corrected for the patient's height. Mean \pm standard deviation was given as the descriptive statistics. Statistical analysis of data was performed by using the IBM software SPSS Statistics 21 for Macintosh. For all volumes except differences of contralateral hemithorax volumes, $\mathrm{P} \leq 0.05$ value was considered statistically significant.

\section{Results}

For both groups, mean age, mean height, and mean weight were found to be $53.25 \pm 14.7$ years, $169.7 \pm 9.4 \mathrm{~cm}$ and $76.9 \pm 13.1 \mathrm{~kg}$, respectively. The lung volume changes were assessed and compared through the CTT images taken before and three months after the treatment. The functional evaluation in the third post-operative/control month was performed with PFT. In the third post-operative/control month, the lung volume was evaluated through control CTTs, and the respiratory volume was evaluated through PFTs. In the surgical stabilization group, the increase in the total lung volume was $36.9 \%$, but it was only $12.9 \%$ in the nonsurgically treated group. A greater volume increase was observed in the surgical stabilization group $(p \leq 0.05)$. In cases where the hemithorax was affected by trauma, the increase was $34.6 \%$ in the surgical stabilization group while it was $10 \%$ in the non-surgically treated group $(\mathrm{p} \leq 0.05)$. In the evaluation of the contralateral hemithorax, it was found that the increases in the stabilization group and in the non-surgical group were $39.2 \%$ and $13.9 \%$ respectively ( $p>0.05$ and Table 2 ).

In the third post-operative/control month, mean FVC, FEV1 and FEV/FVC values were measured at $3227 \pm 1074$, $2690.30 \pm 1080,81.38 \pm 9.7$ in the non-surgical group and $3276 \pm 734,2717 \pm 621,82.91 \pm 6.7$ in the surgical stabilization group, respectively. Although the respiratory volume according to the PFT tests was greater in the surgical stabilization group, the increase was not statistically significant ( $\mathrm{p} \geq 0.05$, Figure 3 ). The mean $\mathrm{CE}$ of the lung volume estimates was 2.02\% (Min: 0.73-Max: 5.44).

Table 2. Estimated lung volumes and their pre- and post-operative differences

\begin{tabular}{|c|c|c|c|c|}
\hline & & $\begin{array}{l}\text { Surgical } \\
\left(\mathrm{cm}^{3}\right)\end{array}$ & $\begin{array}{l}\text { Non- } \\
\text { surgical } \\
\left(\mathrm{cm}^{3}\right)\end{array}$ & P* \\
\hline \multirow[t]{3}{*}{ Trauma side } & Pre-op & $1710.5 \pm 422$ & $1830,6 \pm 422$ & \\
\hline & $\begin{array}{l}\text { Post- } \\
\text { op/control }\end{array}$ & $2303.1 \pm 567$ & $2422.5 \pm 896$ & \\
\hline & Difference & $592.7 \pm 556$ & $197.4 \pm 209$ & 0.04 \\
\hline \multirow[t]{3}{*}{$\begin{array}{l}\text { Contralateral } \\
\text { hemithorax }\end{array}$} & Pre-op & $1739.7 \pm 724$ & $1956.0 \pm 922$ & \\
\hline & $\begin{array}{l}\text { Post- } \\
\text { op/control }\end{array}$ & $2028.0 \pm 612$ & $2229.9 \pm 927$ & \\
\hline & Difference & $682.8 \pm 559$ & $273.9 \pm 315$ & 0.07 \\
\hline \multirow{3}{*}{$\begin{array}{|ll|}\begin{array}{l}\text { Total } \\
\text { volume }\end{array} & \text { lung } \\
\end{array}$} & Pre-op & $3450.2 \pm 934$ & $3786.6 \pm 1258$ & \\
\hline & $\begin{array}{l}\text { Post- } \\
\text { op/control }\end{array}$ & $4725.6 \pm 140$ & $4257.9 \pm 1476$ & \\
\hline & Difference & $1275.4 \pm 100$ & $471.4 \pm 392$ & 0.02 \\
\hline
\end{tabular}

Pre-op: pre-operative, Post-op: post-operative *p values obtained via ANCOVA 

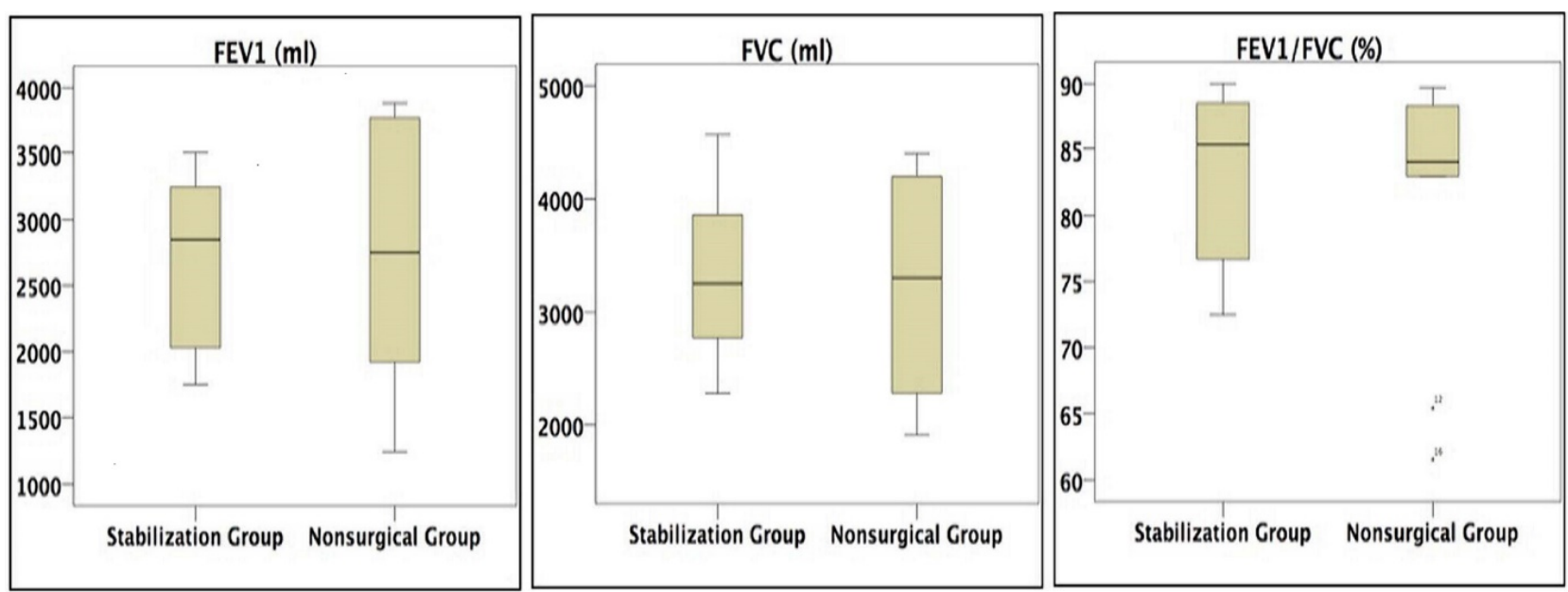

Fig. 3. Delineation of the lung boundaries during inspiration to measure the surface areas of the lung volume using planimetry method in a patient. A: CCT section of the patient in admission. B: CCT section of the same patient in the third post-operative month.

\section{Discussion}

The standard treatment method for patients with flail chest is the conservative treatment, which consists of bronchial drainage, selective antibiotic therapy, pain control, and selective mechanical ventilation. The main aim of this treatment is to ensure effective ventilation and to prevent progressive parenchymal damage, together with complications and sequelae that may occur (Lafferty et al., 2011; Fokin et al., 2020). However, the complications which develop due to prolonged ventilation support increase the rates of morbidity and mortality and cause prolonged hospital stays (Mohr et al., 2007; Beks et al., 2019)

In patients who do not need ventilation, pain reduction and comfortable cough are ensured with the stabilization of the chest wall by the use of fixation bandages. However, this method prevents adequate expansion of the thorax and causes atelectasis and consequently respiratory failure. Additionally, the spontaneous healing of the displaced rib fractures can cause chest wall deformities in the late period, which can cause lost volume and atelectasis in the underlying lung, consequently leading to impairments in pulmonary function in the chronic phase (Peek et al., 2019).

In recent years, it has been reported that surgical stabilization methods performed on selected patients shortens the ventilation and intensive care follow-up period and provides reduction in the rates of ventilation-related morbidity and mortality (Nirula et al., 2009; Lin et al., 2016) Furthermore, the chest wall deformities that can develop in the chronic period and the volume decrease in the chest wall secondary to the deformity can be prevented by chest wall stabilization. This thesis is supported by the fact that in our study, the lung volumes assessed in the third month of the post-operative period were significantly greater in patients who underwent stabilization compared to those who did not undergo stabilization.

Main indications for the surgical stabilization in flail chest patients in the early period are respiratory failure development despite aggressive medical treatment, a large flail chest covering the anterolateral chest wall, and a thoracotomy performed due to another complication (Lin et al.,2016). In our series, one patient had chronic pain and non-union rib fractures, one had undergone thoracotomy due to intrathoracic hematoma drainage and decortication, and the remainder had a large flail chest area which required mechanical ventilation support and/or which was located in the anterolateral area and caused paradoxical respiration. Chronic pain and permanent chest wall deformities in the chronic phase of flail chest are the most frequent indications for surgical stabilization (Pieracci et al., 2017)

In our series, only one patient underwent stabilization in the chronic period; the rest underwent stabilization in the early post-traumatic period (within the first five days), due to the indications mentioned. The main aim of stabilizations performed at this stage is to elevate the collapsed segment and to restore the defect, thus preventing the chest wall deformities which might occur in the chronic phase (Pieracci, 2019). In all patients, the aim was to restore the depressed segment which might cause paradoxical respiration in the acute period and restriction and volume loss in the thorax wall in the chronic period. In this approach, chest wall stabilization was achieved in all 10 patients, and no secondary dislocation and other complications were encountered during the third month follow-up. Granetzny et al. (2005) emphasized in their study that the rate of success for chest wall stabilization was $85 \%$ in patients who underwent stabilization due to flail chest.

There must be adequate space in the thorax for the lung to expand. A decrease in the volume of lung is inevitable during the healing of displaced rib fractures; consequently, a decrease in the lung volume is also an expected result Tanaka et al.,2002; Sengul et al., 2013). Accordingly, the restoration of chest wall integrity in the early period may not only shorten the duration of mechanical ventilation but also keep the chest wall volume within normal limits, thus increasing lung volume (Athanassiadi et al., 2004). However, it may not be possible to measure the lung volume changes and PFTs independently and accurately due to patient-related factors, 
particularly in the early post-traumatic period. Similarly, in our study, although lung increases or decreases in the early post-traumatic period were measured independently and accurately on the tomographic images with stereological methods, the results did not correlate with PFTs.

In our previous studies, it has been proven that the remaining lung after lung resection has expansion capacity. The volume changes in the lung were identified using unbiased techniques through CTT images (Başoglu et al., 2007) Computed thorax tomography can distinguish lung tissue from other soft tissues very well and can easily identify the margins of the tissue; therefore, it is very useful for stereological studies because the changes in the volumes of thorax and lung due to displaced fractures can easily be assessed independently of the patient-related variables (Başoğlu et al., 2007). In our study, assessments of lung volumes were planned to be performed via tomographic sections. Rib fractures in flail chest patients are often accompanied with pneumothorax and/or hemothorax, and this situation may cause problems in determining the real margins of the lung. Thus, the measurement of lung volume was considered to be a more realistic assessment method. The measurements performed in the third post-operative month revealed that total lung volume increased by $39.8 \%$ in patients who underwent stabilisation, but in patients who were treated with non-surgical methods, this rate was only $12.3 \%$.

In the chronic phase, the majority of these patients develop chest wall deformities and restrictive-type pulmonary function disorders due to pain (Wanek et al., 2004, Fabbri et al.,2009). Lardinois et al. (2000) and Granetzny et al. (2005) have reported in their studies that restrictive-type respiratory failure was prevented in patients who underwent surgical fixation in the early period, and that according to the pulmonary function test performed in the sixth post-operative month, the total lung volumes of these patients were significantly greater than those of conservatively treated patients. However, a PFT performed within the first six months of fixation may not always reflect reality because of comorbid traumas and secondary pulmonary diseases. In their studies found that FVC, FEV, TLC, and PEF values of flail chest patients treated conservatively were lower in the second month of the followup. We also determined a minimal difference in FVC, FEV1, and FEV1/FVC values of the patients who underwent surgical rib stabilisation compared to the other group. However, these values did not correlate with the volume increases determined with stereological methods through thorax CTs. On the other hand, in a study conducted in our clinic on patients with chest wall deformity, it was determined that when stereological assessments were compared, post-operative lung volumes were $12 \%$ greater than the pre-operative lung volumes, but the result did not correlate with the PFTs. Post-operative pain, comorbid traumas, and secondary pulmonary diseases are thought to be the most likely reasons for this inconsistency (Wanek, 2004). Lardinois et al. (2000) and Granetzny et al.
(2005). We believe that uncorrelated PFT results and the results of lung volume changes were mainly related to the pain caused by the broken ribs, and to ineffective bronchial cleaning in the recovery period and the comorbid pulmonary contusion. Additionally, ensuring the normal chest wall configuration with stabilization in the early period will contribute a great deal to PFT recovery in the chronic period when the pulmonary contusion and the above-mentioned negative factors decrease.

In conclusion, surgical chest wall stabilization could be a primary choice of treatment in flail chest patients with extremely displaced rib fractures that can cause chest wall volume decreases and chest wall deformities. Restrictive respiratory dysfunctions can be prevented with an increase in chest wall volume and lung volume. However, we believe that studies with larger patient groups which will be followed for longer periods are needed to evaluate the long-term effects of this therapeutic approach.

\section{Acknowledgment}

None

\section{Conflict of Interest}

None

\section{References}

1. Ahmed, Z., Mohyuddin, Z., 1995. Management of flail chest injury: internal fixation vs. endotracheal intubation and ventilation. J. Thoracic. Cardiovasc. Surg. 110:1676-1680.

2. Althausen, P.L., Shannon, S., Watts, C., Thomas, K., Bain, M.A., Coll, D., O'mara, T.J., Bray, T.J., 2011. Early surgical stabilization of flail chest with locked plate fixation. J. Orthop. Trauma. 11, 641-7.

3. Athanassiadi, K., Gerazounis, M., Theakos, N. 2004. Management of 150 flail chest injuries: analysis of risk factors affecting outcome. Eur. J. Cardiothorac. Surg. 26:373-376.

4. Basoglu, A., Buyukkarabacak, Y., Sahin, B., Kaplan, S., 2007. Volumetric evaluation of the lung expansion following resection: a stereological study. Eur. J. Cardiothorac. Surg. 31:512-7

5. Beks, R.B., Peek, J., de Jong, M.B., Wessem ,K.J.P., Öner, C.F., Hietbrink, F., Leenen, L.P.H., Groenwold, R.H.H., Houwert, R.M. 2019. Fixation of flail chest or multiple rib fractures: current evidence and how to proceed. A systematic review and metaanalysis. Eur. J. Trauma. Emerg. Surg. 45, 631-644.

6. Bulger, E.M., Arneson, M.A., Mock, C.N., Jurkovich, G.J., 2000. Rib fractures in the elderly. J. Trauma. 48:1040-7.

7. Fabbri, C., Mazieri, M., Cirocchi, R., Bisacci, R., Cagini, L., 1996. Flail chest. Minerva. Chir. 51:669-673.

8. Granetzny, A, Abd El-Aal, M., Emam, E., Shalaby, A., Boseila, A., 2005. Surgical versus conservative treatment of flail chest: evaluation of the pulmonary status. Interact. Cardiovasc. Thorac. Surg. 4:583-587.

9. Fokin, A.A., Hus, N., Wycech, J., Rodriguez, E., Puente, I.,2020. Surgical stabilization of rib fractures: 1ndications, techniques, and pitfalls. JBJS. Essent. Surg. Tech.7;10(2):e0032.

10. Konstantinov, I.E., Saxena, P., Wood, D.J., 2009. Stabilisation of chronic flail chest: a novel approach of surgical fixation and osteogenesis. Thorax. 64:265-266. 
11. Lafferty, P.M., Anavian, J., Will, R.E., Cole, P.A., 2011. Operative treatment of chest wall injuries: indications, technique, and outcomes. J. Bone. Joint. Surg. Am. 5, 93(1):97-110.

12. Lardinois, D., Krueger, T., Dusmet, M., 2000. Pulmonary function testing after operative stabilisation of the chest wall for flail chest. Eur. J. Cardiothorac. Surg. 20:496-501.

13. Lin., F.C.F., Li, R.Y., Tung, Y.W., Jeng, K.C., Tsai, S.C.S., 2016. Morbidity, mortality, associated injuries, and management of traumatic rib fractures. J. Chin. Med. Assoc. 79(6):329-34

14. Mazonakis, M., Sahin, B., Pagonidis, K., Damilakis, J., 2011. Assessment of Left Ventricular Function and Mass by MR Imaging: A Stereological Study Based on the Systematic Slice Sampling Procedure. Acad. Radiol. 18(6):738-744.

15. Mehrabi, N.F., Singh-Bains, M.K., Waldvogel, H.J., Faull, R.L.M. 2018. Stereological methods to quantify cell loss in the huntington's disease human brain.Methods. Mol. Biol. 1780:1-16.

16. Mohr, M., Abrams, E., Engel, C., Long, W.B., Bottlang, M., 2007. Geometry of human ribs pertinent to orthopedic chest wall reconstruction. J. Biomech. 1310-1317.

17. Nirula, R., Diaz, J.J. Jr, Trunkey, D.D., Mayberry, J.C., 2009. Rib fracture repair: indications, technical issues, and future directions. World. J. Surg. 33:14-22.

18. Peek, J.., Beks, R.B., Kremo, V., van Veelen, N., Leiser, A., Houwert, R.M., Link, B.C., Knobe, M., Babst, R.H., Beeres, F.J.P. 2019. The evaluation of pulmonary function after rib fixation for multiple rib fractures and flail chest: a retrospective study and systematic review of the current evidence. Eur. J. Trauma. Emerg. Surg. Nov 25.

19. Pieracci, F.M., 2019. Completely thoracoscopic surgical stabilization of rib fractures: can it be done and is it worth it? J.
Thorac. Dis. 11(Suppl 8): S1061-S1069.

Pieracci, F.M., Majercik, S., Ali-Osman, F., Ang, D., Doben, A., Edwards, J.G., French, B., Gasparri, M., Marasco, S., Minshall, C., Sarani, B., Tisol, W., VanBoerum, D.H., White, T.W., 2017. Consensus statement: Surgical stabilization of rib fractures rib fracture colloquium clinical practice guidelines. Injury. 48(2):307-321.

20. Sahin, B., Alper, T., Kökçü, A., Malatyalioglu, E., Kosif, R., 2003. Estimation of the amniotic fluid volume using the Cavalieri method on ultrasound images. Int. J. Gynaecol. Obstet. 82:25-30

21. Sahin, B., Emirzeoglu, M., Uzun, A., Incesu, L., Bek, Y., Bilgic, S., Kaplan, S., 2003. Unbiased estimation of the liver volume by the Cavalieri principle using magnetic resonance images. Eur. J. Radiol. 47:164-170.

22. Sahin, B., Ergur, H., 2006. Assessment of the optimum section thickness for the estimation of liver volume using magnetic resonance images: A stereological gold standard study. Eur. J. Radiol. 57(1):96-101.

23. Sengul, A.T., Sahin, B., Celenk, C., Basoglu, A. 2013. Postoperative lung volume change depending on the resected lobe. Thorac. Cardiovasc. Surg. 6:131-137.

24. Tanaka, H., Yukioka, T., Yamaguti, Y., Shimizu, S., Goto, H., Matsuda, H., Shimazaki, S., 2002. Surgical stabilization of internal pneumatic stabilization? A prospective randomized study of management of severe flail chest patients. J. Trauma. 52:72732.

25. Wanek, S., Mayberry, J.C., 2004. Blunt thoracic trauma: flail chest, pulmonary contusion, and blast injury. Critical. Care. Clinics. 20:71-81. 